Rhode Island College

Digital Commons @ RIC

$1-1-2012$

\title{
Quality Management of Chemotherapy: Induced Nausea and Vomiting with Minimal Constipation
}

Mary Ellen Danzer
Rhode Island College

Follow this and additional works at: https://digitalcommons.ric.edu/etd

Part of the Geriatric Nursing Commons

\section{Recommended Citation}

Danzer, Mary Ellen, "Quality Management of Chemotherapy: Induced Nausea and Vomiting with Minimal Constipation" (2012). Master's Theses, Dissertations, Graduate Research and Major Papers Overview. 198.

https://digitalcommons.ric.edu/etd/198

This Major Paper is brought to you for free and open access by the Master's Theses, Dissertations, Graduate Research and Major Papers at Digital Commons @ RIC. It has been accepted for inclusion in Master's Theses, Dissertations, Graduate Research and Major Papers Overview by an authorized administrator of Digital Commons @ RIC. For more information, please contact digitalcommons@ric.edu. 
QUALITY MANAGEMENT OF CHEMOTHERAPY- INDUCED NAUSEA AND VOMITING WITH MINIMAL CONSTIPATION

A Major Paper Presented

By

Mary Ellen Danzer

Approved:

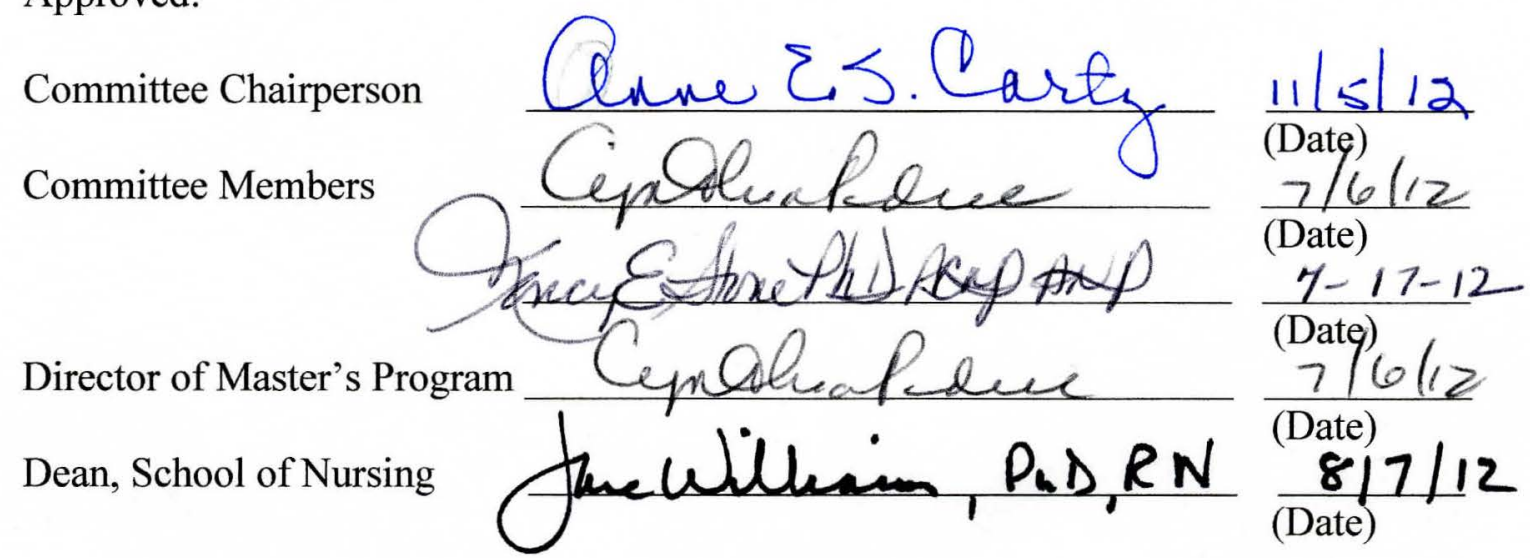


QUALITY MANAGEMENT OF CHEMOTHERAPY- INDUCED NAUSEA AND

VOMITING WITH MINIMAL CONSTIPATION

by

Mary Ellen Danzer

A Major Paper Submitted in Partial Fulfillment

of the Requirements for the Degree of

Master of Science in Nursing

in

The School of Nursing

Rhode Island College

2012 


\begin{abstract}
Although clients diagnosed with colorectal cancer (CRC) are receiving more advanced and successful chemotherapy treatments, the side effects of these treatments continue to cause anxiety, discomfort, pain, and a diminished quality of life. Treatments must be developed to avoid some of the most unpleasant symptoms a client experiences as a result of these treatments. The purpose of this study was to examine the incidence of constipation related to the treatment of chemotherapy-induced nausea and vomiting (CINV) and to identify treatments that are successful in managing constipation while maintaining effective control of CINV. Constipation can be a significant problem with a negative impact on lifestyle during chemotherapy and needs aggressive management. Use of ondansetron as an antiemetic is associated with the development of constipation. A retrospective study was performed to identify the rate of constipation among a cohort of CRC clients and to determine effective treatment of constipation in the CRC client undergoing chemotherapy. Results of the study include increased rate of constipation in the study group, and limited documentation of symptoms and treatments. The study provided evidence that constipation is a significant side effect of CINV treatment with ondansetron and requires a comprehensive history to determine etiology. Communication is an important aspect of care, with the Clinical Nurse Specialist (CNS) informing staff of client history and insuring continuity of care. Implications and recommendations are identified and discussed.
\end{abstract}




\section{Acknowledgements}

I am heartily thankful to my advisors, Cynthia Padula, PhD and Anne Carty, $\mathrm{DNSc}, \mathrm{RN}$ whose direction, guidance and support from the initial draft to the final version enabled me to develop an understanding of the project.

I am indebted to my student colleagues for providing a supportive and fun environment in which to learn and grow.

A special mention to my friend Nadine for her direction and support without which the completion of the paper would not have been possible.

I am most grateful to my husband, Ken for his support and great understanding at all times.

Last, by but no means least, I thank my children, family and friends for their support and encouragement throughout this process. 
Table of Contents

Table of Contents....................................................

Background and Problem Statement..........................................

Literature Review....................................................4

The Impact of the Clinical Nurse Specialist on Management of Symptoms of

Constipation............................................................... 13

Theoretical Framework.....................................................16

Method..................................................................20

Results...............................................................23

Summary and Conclusions...............................................26

Implications and Recommendations....................................29

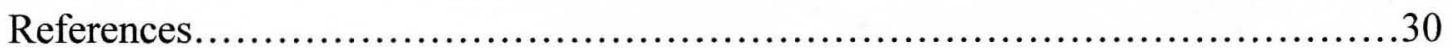


Maintaining Quality Management of Chemotherapy-Induced Nausea and

\section{Vomiting with Minimal Constipation \\ Background and Problem Statement}

Oncology cancer treatments have evolved over the last 30 years. Clients are now interpreting a cancer diagnosis as a chronic disease rather than an end of life experience. Approximately $55 \%$ of cancer patients survive five years, meaning that many clients become long-term survivors, a phenomenon made possible by the development of new therapies (Jefford et al., 2011). CINV is a serious problem associated with poor healthrelated quality of life (Likun, Xiang, Yi, Xin, \&Tao, 2011). Approximately $70-80 \%$ of cancer clients receiving chemotherapy experience nausea, vomiting, or both symptoms (Rangwala, Zafar, \& Abernethy, 2012). Frequently, when clients are interviewed just prior to beginning therapy, the one fear commonly identified, second only to hair loss, is the fear of feeling sick and vomiting (Likun et al., 2011). Anti-nausea medications have provided much relief from the undesirable chemotherapy side effects: nausea and vomiting. The development of a new classification of drugs, the 5-HT3 inhibitors, to manage CINV has given many oncology clients the opportunity to receive the most effective treatment with minimal side effects. The use of this highly effective antiemetic therapy has allowed administration of chemotherapy in the ambulatory, outpatient setting (Lohr, 2008). As with any medication, there can be undesirable side effects. One very disrupting, and at times painful, side effect of the 5HT-3 medications is constipation. The clinician must consider the client's presenting symptoms and the differential diagnosis of constipation when the client is receiving chemotherapy treatment. 
Constipation is a distressing condition for cancer patients and is frequently under diagnosed (Nagaviroj, Yong, Fassbender, Zhu, \& Onechuk, 2011). Constipation can be more distressing to clients than pain and can cause some clients to decline further treatments (Rangwala et al., 2012). Constipation is usually caused by a combination of poor oral intake, decreased physical activity, and drugs that have a neuropathic effect such as opiate pain medications, antiemetic medications, antidepressants, antihypertensive medications, and some chemotherapeutic agents (Rangwala et al., 2012). These agents are important contributors to constipation because they slow intestinal transit time (Shafi \& Bresalier, 2010). In clients who have a more advanced colorectal cancer, constipation may be suggestive of disease progression and may lead to life-threatening complications (Farjardo, Cremonini, \& Talley, 2006). Other gastrointestinal abnormalities need to be ruled out prior to diagnosing constipation as due to treatment for CINV. Electrolyte imbalances and other reversible causes should be treated. Drugs contributing to constipation need to be avoided if they are not necessary for effective treatment of the client's problem. The impact of the symptoms of constipation on the quality of life of a cancer client is often greater than for those not affected by the disease. The oncology community frequently affected by this undesirable side effect is the CRC clientele. Colorectal clients are vulnerable to constipation secondary to necessary treatment, and this symptom is particularly problematic in this group (Farjardo et al., 2006).

Clients with $\mathrm{CRC}$ are generally treated with moderately emetogenic therapies, which require the use of anti-nausea medications. Many clients also need to take 
narcotics for pain; both contribute to constipation which is painful, decreases quality of life (QOL), and results in/leads to weight loss and depression (Likun et al., 2011). Recognition of concomitant psychological stressors should be considered as part of the client's plan of care (POC) (Farjardo et al., 2006). Assuring that the symptom of constipation is prevented when possible and/or effectively managed is an important nursing intervention that has been understudied in this population (Farjardo et al., 2006). 


\section{Literature Review}

A systematic review of the literature was performed using PubMed, CINAHL and OVID databases. Internet sites relevant to this review included www.American Cancer Society, Up-To-Date and cancer.org. Keywords searched included: cancer, hair loss, constipation, chronic disease, life expectancy, chemotherapy, nausea, vomiting, emesis, colon cancer, rectal cancer, CRC, and nursing. Search terms focused on constipation in CRC undergoing treatment with moderately emetogentic chemotherapy and constipation. By combining several keywords, the literature search was more concise yielding more specific articles. Literature will focus on CRC, chemotherapy, chemotherapy-induced side effects, constipation, and constipation in clients with CRC, antiemetic treatment, and their impact on the role of the CNS.

\section{Colorectal Cancer: Background and Epidemiology}

In 2007, The National Program of Cancer Registries identified CRC as the $4^{\text {th }}$ most commonly diagnosed cancer in Rhode Island (Cancer.org). In 2011, the American Cancer Society (ACS) ranked CRC as the third most frequently diagnosed cancer and third leading cause of cancer death in both men and women in the United States.

Research has demonstrated that CRC "arises as a consequence of the accumulation of genetic and heritable changes in gene expressions that drive the transformation and progression of normal colonic epithelial cells to cancer" (Saif \& Chu, 2010). This transformation is known as tumorigenesis. Tumorigenesis is the term used to describe the time it takes for a polyp to transform into true cancer, with the average being eight to 12 years (Saif \& Chu, 2010). Polyps are non-cancerous growths in the rectum and colon. 
Though most polyps will not become cancerous, detecting them and removing them can actually prevent cancer from occurring (ACS). The ACS (2011) recommends a colonoscopy be performed by the age of 50, as most CRCs develop from adenomatous polyps. Screening before age 50 is recommended for those at increased risk of developing CRC. Those included in this population are persons with a family history of CRC or certain medical conditions (ACS). The National Comprehensive Cancer Network (NCCN) Guidelines (2011) suggest that children of clients with CRC have their initial screening colonoscopy ten years prior to the age the client is at time of diagnosis. In other words, if the client was 43 years of age at the time of diagnosis, their children should be screened when they are 33 years of age.

The ACS estimated that in 2011, approximately 141,210 people would be diagnosed with CRC, and 49,380 would die from the disease in the US. CRC incidence rates have been declining since the mid 1980 's, with a reported decline of $3 \%$ in men and $2.3 \%$ in women. This decline has been largely due to the detection and removal of precancerous lesions as a result of colorectal screening (ACS, 2011).

While rates have been declining among adults 50 years and older, the incidence of $\mathrm{CRC}$ has increased among adults younger than 50 years. The reasons for this increase are not entirely known but may reflect increasing trends in obesity and and/or unfavorable dietary patterns in children and young adults (ACS, 2011). Up to $25 \%$ of clients may have a personal or family history suggestive of an inherited syndrome (Pollock \& Welsh, 2011). 


\section{Colorectal Cancer: Treatment Overview}

Most people with colon cancer, especially in the early stage, are treated with surgery to remove the tumor (ACS, 2011). Rectal cancer is treated with surgery only, unless the cancer has spread to other areas. Combination radiation and chemotherapy are given to those rectal cancer clients with a risk of recurrence or known to have the cancer in other areas of the body. The ACS describes cancer surgery as the removal of a cancerous tumor. When a section of the colon or rectum is removed, the surgeon can usually reconnect the healthy parts, allowing the client to eliminate normally. For some clients, this connection is not possible and they will require a colostomy. A colostomy is formed when the surgeon makes an opening or stoma in the skin of the abdomen for waste to leave the body. A bag is utilized to collect the waste material. This bag has an adhesive backing, which makes the appliance adhere to the skin. According to the ACS (2011), most clients who need a colostomy need it only temporarily to allow the colon or rectum to heal. After healing takes place, in approximately 6-8 weeks, the surgeon will reconnect the parts of the intestine and close the stoma.

\section{Chemotherapy}

Adjuvant chemotherapy (chemotherapy after surgery) or radiation may also be given. The chemotherapy drugs most often used for the treatment of $\mathrm{CRC}$ are Fluorouracil, Oxaliplatin, Leucovorin and Irinotecan (Arcangelo \& Peterson, 2006). Chemotherapy affects the cancer cells but also affects some cells normally found in the body. There are several side effects commonly experienced after receiving these chemotherapeutic agents. Side effects depend on the type of drug, the duration of 
treatment, the amount taken and whether or not the treatment spans several days (Arcangelo \& Peterson, 2006). Fluorouracil is used before and after surgery. Expected side effects include diarrhea, mouth sores, difficulty swallowing, poor appetite, decreased blood cell production, pain, and redness and blistering of the palms and soles of the feet. Oxaliplatin is used after surgery or in the case of metastatic disease. One very specific side effect clients experience is cold sensitivity or neuropathy. This side effect prevents clients from eating or drinking cold fluids, and/or frozen foods for a minimum of four days following treatment. Other side effects include decreased proprioception (the body's sense of movement and position), nausea, vomiting, diarrhea, and decreased blood cell production. Irinotecan is used in clients with metastatic CRC and can cause severe diarrhea and abdominal cramping. Leucovorin is folic acid, which enhances the 5fluorouracil (Up-To-Date). The ACS (2011) recommends treatment decisions be made collaboratively by the physician and client. Physician and client must consider the best options for the stage of the cancer and location of the cancer as well as the risks and benefits of each treatment.

\section{Chemotherapy - Induced Side Effects}

Chemotherapy induces a wide array of side effects. For purposes of this literature review, only those side effects specific to the GI tract will be discussed. CINV is a very common side effect and is associated with a significant deterioration in QOL with impaired functional abilities (Rangwala et al., 2012). The greatest impact on QOL occurs in clients with the poorest control of CINV (Rangwala et al., 2012). Likun et al. (2011) surveyed clients who experienced more than two episodes of CINV. These clients stated 
that nausea and vomiting were the most feared effects of the chemotherapy. The experience of CINV puts these clients at risk for serious metabolic disturbances, malnutrition, weight loss, depression, and increased risk of not completing treatment (Rangwala et al., 2012). The likelihood of CINV is dependent on the dose of the drug and the emetogenicity of the chemotherapeutic agents. The NCCN Guidelines (2011) define emetogenicity as the potential risk that a client will experience nausea and/or vomiting as a result of receiving this treatment. Repeated chemotherapy cycles and client risk factors significantly influence CINV.

According to the NCCN Guidelines (2011), clients receiving moderately emetogenic chemotherapy are at risk for both acute and delayed nausea/vomiting. New classifications of drugs to manage these problematic symptoms include the Serotonin 5HT3 receptor antagonists plus dexamethasone. These drugs block the chemotherapy trigger zone in the brain, decreasing the chance individuals will experience CINV. Some of these drugs are Ondansetron, Palonosetronin, and Aloxi.

Antiemetic therapy should be initiated before chemotherapy is begun and should be continued for the same length of time as the duration of the emetic activity of the chemotherapeutic agent being used (NCCN Guidelines, 2011). In other words, the client receives antiemetic therapy prior to infusion of chemotherapy and receives antiemetic therapy for as long as the chemotherapy is causing the symptom. The chemotherapies generally administered to the client with CRC cause nausea for up to 3-5 days post treatment; thus antiemetic treatment for this side effect should last at least that long. 
These drugs have significantly improved the control of acute CINV, but delayed CINV remains a significant clinical problem (Navari, 2009).

The practitioner must decide which antiemetic drug is best for each client. If CINV is not treated, nausea can significantly affect a client's quality of life, leading to poor compliance with further chemotherapy. Additionally, nausea and vomiting can result in metabolic imbalances, degeneration of the client's performance status and mental status, wound dehiscence (the unexpected opening of an incision), and esophageal tears (NCCN Guidelines, 2011).

One potential side effect of this class of drugs is constipation, a particularly problematic issue for clients with CRC. Use of these anti-nausea medications leads to increased rates of constipation; there is an $18-20 \%$ chance of constipation when $5-\mathrm{HT} 3$ inhibitors are prescribed (Farjardo et al., 2006). Ondansetron is the drug of choice in CRC, and the associated risk of constipation is reported to be $18 \%-20 \%$ (Up-to-Date). The unpleasant symptom of constipation can significantly compound the physical and emotional discomfort experienced by clients.

\section{Constipation: Definition and Impact}

Constipation is a general term used to describe the difficulties that a client experiences with moving his or her bowels. There is no universally accepted definition of constipation (Heitkemper \& Wolff, 2007). Healthcare providers typically define constipation as stool frequency of less than three bowel movements per week. A thorough history from the client is necessary as clients define constipation as straining, hard stool, feeling of incomplete evacuation, and nonproductive urgency (Rao \& Go, 
2010). In addition to the thorough history, the practitioner performs an abdominal exam and digital rectal exam (Heitkemper \& Wolff, 2007). Evidence in both diseasespecific and generic QOL instruments has shown that constipation is associated with impaired health-related QOL (Rao \& Go, 2010). Clients reported lower scores for physical functioning, mental health, general health perception, and bodily pain when compared to clients with no constipation. Additionally, constipation is a significant driver of healthcare costs, being ranked among the top five most common physician diagnoses for gastrointestinal outpatient visits (Rao \& Go, 2010). Constipation is a condition the clinician must take into consideration when evaluating clients with advanced illness (Librach, Bouvette, DeAngelis \& Pereira, 2010).

The goal of managing clients with constipation is not only to relieve the constipated state but also to prevent recurrence (Heitkemper \& Wolff, 2007). Educating the client to increase fluid intake, exercise, and add fiber to the diet in addition to taking a stimulant laxative is recommended. The laxative is for short-term use only. Finding the cause of the constipation and eliminating it are the best options. There are no official guidelines dictating the order in which treatment options for clients with constipation should be administered. Despite the strides in evidence-based nursing, numerous gaps remain (Heitkemper \& Wolff, 2007). Managing constipation in clients with CRC is an important nursing concern.

\section{Constipation in Clients with Colorectal Cancer}

Constipation is a problem of increased significance for clients with cancer in general, and for those with CRC in particular. A study by Wilson, Alexander, Kind, \& 
Phil (2006) that examined QOL issues in the CRC client, found that $21.9 \%$ reported problems with constipation. Constipation estimates in the general population range from $2 \%$ to $28 \%$, whereas $40 \%$ of the palliative care population has reported symptoms of constipation (Wilson et al., 2006). The use of other medications such as pain medications contributes to the increased rates of constipation. Constipation has a demonstrated negative impact on QOL but improved treatment may lessen the negative impact on a client's life (Farjardo et al., 2006).

$\mathrm{CRC}$ clients are at increased risk of developing constipation as well as other side effects. A comprehensive medical history and physical exam are vital components in the evaluation of clients who present with constipation (Fajardo et al., 2006). Determining the change in the pattern and frequency of bowel movements, stool consistency, and ease of stool passage provides some indication of the severity of constipation. The nurse can assist the client with recognizing early symptoms of constipation and, more importantly, proactively anticipate constipation when certain medications are given to treat either the cancer or the side effects of chemotherapy treatments (Farjardo et al., 2006). Of note, bulk laxatives are of limited use in the cancer client who often has difficulty maintaining an adequate volume of fluid and may exacerbate opiate-induced constipation (Farjardo et al., 2006).

Reviewing all medications taken and educating clients about medications that would effectively treat constipation should occur at start of treatments and at each subsequent visit. It is essential to review the client's entire medication list during each visit, as this can help identify those medications, which could potentially cause 
constipation, and knowing what medications the client is taking helps to avoid some side effects. The CNS needs to listen to client, analyze the challenges the oncology client faces when a particular symptom arises, and implement the appropriate treatment plan (Miakowski, 2011). 
The Impact of the CNS on Management of Symptoms of Constipation

The dimensions of the CNS role are expert clinician, consultant, educator, and researcher. The CNS cares for and improves outcomes of clients through three spheres of influence: the client/family, nursing/nursing practice, and organizational/systems sphere (McKinley, 2007). Direct clinical practice is central to the care of any complex or unique client. Each client is unique and may display numerous problems. A CNS working with clients with cancer would demonstrate competence in the care of the complex oncology client. The CNS would identify client problems, identify staff learning needs, and assist with developing a plan of care (POC) to address complex issues. Through clinical leadership and influence, the CNS implements strategies for change designed to improve client care (Client sphere) positively impacts nursing practice (Nursing/Nursing Practice sphere), and directly impacts the care of patients overall (Organization/Systems sphere) (McKinley, 2007). A study by Kim (2011) compared outcomes including pain, fatigue, anxiety, and satisfaction, health related-quality of life (HRQOL), ease of access, and unexpected emergency room visits for patients with cancer in South Korea who were cared for by a CNS with clients with cancer who were not cared for by a CNS. No significant effects were observed on anxiety from of unexpected emergency room visits. CNS interventions were found to diminish some intensity scores of pain and fatigue, and to increase HRQOL, satisfaction with trustworthiness, and ease of access (Kim, 2011). An important role of the CNS working with clients undergoing chemotherapy is to provide maximal protection against CINV. The CNS collaborates with nurses, physicians, and other members of the health care team and has a good understanding of 
the knowledge and skills of other health care team members (McKinley, 2007). The CNS plays a key role in the liaison work with members of the multidisciplinary team as well as care providers in the community of referring agencies (Andreyev, Davidson, Gillespie, Allum, \& Swarbrick, 2012). Effective collaboration is the result of clinical competence, effective communication, mutual trust, collegiality, and a favorable organizational structure (Hanson \& Spross, 2005).

There have been many changes in the management of CINV over the last two decades and nurses and clinicians caring for the clients receiving chemotherapy have several classes of agents to choose from when ordering anti-emetics (Viale, 2006). The development of the 5-HT3 category of medications represents a significant advance in antiemetic therapy. These agents have been shown to be effective in controlling the acute nausea and/or vomiting associated with cancer chemotherapy (NCCN Guidelines, 2011). It is difficult to recommend a specific antiemetic regimen for each client. The CNS evaluates the effectiveness of the drug for each regimen. The CNS must then educate the client about expected side effects and direct the client in the appropriate management of these side effects (Fulton, Lyon, \& Goudreau, 2010).

When choosing a 5HT3, thorough assessment, including a history of constipation, must be considered especially in the $\mathrm{CRC}$ population. The ability to engage in rational and deliberate reasoning or clinical inquiry to effectively solve clinical problems in the nursing domain is one of the cornerstones of the CNS (Fulton et al., 2010). The CNS is challenged to assess clients for signs and symptoms of constipation by overcoming communication barriers associated with discussion of bowel habits (Heitkemper \& Wolff, 
2007). Characteristics, such as age, ethnicity, and co-morbidities, should be considered because they may affect the presentation and/or treatment of symptoms. This type of approach communicates to the clients and their families that symptoms are important (Sousa, McDermott, \& Weiss, 2011). Many clients are sensitive about discussing issues pertaining to bowel habits. Asking targeted questions pertaining to bowel habits and related symptoms helps generate dialogue and establish how the client defines constipation (Heitkemper \& Wolff, 2007). Laxatives should be routinely started in clients predisposed to constipation (Rangwala et al, 2012). Rangwala recommends that clients should increase fluids and physical activity and those clinicians need to consider reversible causes of constipation such as concurrent use of multiple constipating medications or metabolic abnormalities (e.g hypercalcemia). Effective CNS practice requires a highly developed perspective that puts the nursing care of the client in the foreground of clinical reasoning, with medical problems and disease focused care as critical background context (Fulton et al., 2010).

The goal of this project was to examine the incidence of constipation in clients with colorectal cancer on 5-HT3 inhibitors and to identify treatments that are successful in managing constipation while maintaining effective control of CINV. Next, the framework used to guide this proposed research will be discussed. 


\section{Theoretical Framework}

\section{Theory of Unpleasant Symptoms}

Nursing literature abounds with the admonition that knowledge development requires strong links among theory, research, and practice. Research-practice linkages have been more firmly established than those of nursing theory to research and practice (Lenz, Suppa, Gift, Pugh, \& Milligan, 1995). The Theory of Unpleasant Symptoms (Figure 1) represents a theory developed for practice from practice-based research. The Theory of Unpleasant Symptoms specifies that the experience of symptoms is multidimensional and incorporates the potential interactions of multiple symptoms (Sousa, et al., 2011).

The Theory of Unpleasant Symptoms (Lenz et al., 1995) was initially published in 1995 and then updated in 1997 . The theory is based on the premise that there are commonalities in the experiences of different symptoms among different groups and in different situations. The theory was developed to integrate existing knowledge about a variety of symptoms to better prepare the nurses in symptom management (Lenz, Suppa, Gift, Pugh, \& Mulligan, 1997). The Theory of Unpleasant Symptoms is a middle-range theory that can guide research and practice across multiple clinical populations. The Theory of Unpleasant Symptoms was proposed as a means of integrating information about a variety of symptoms and was developed through the collaborative effort of 


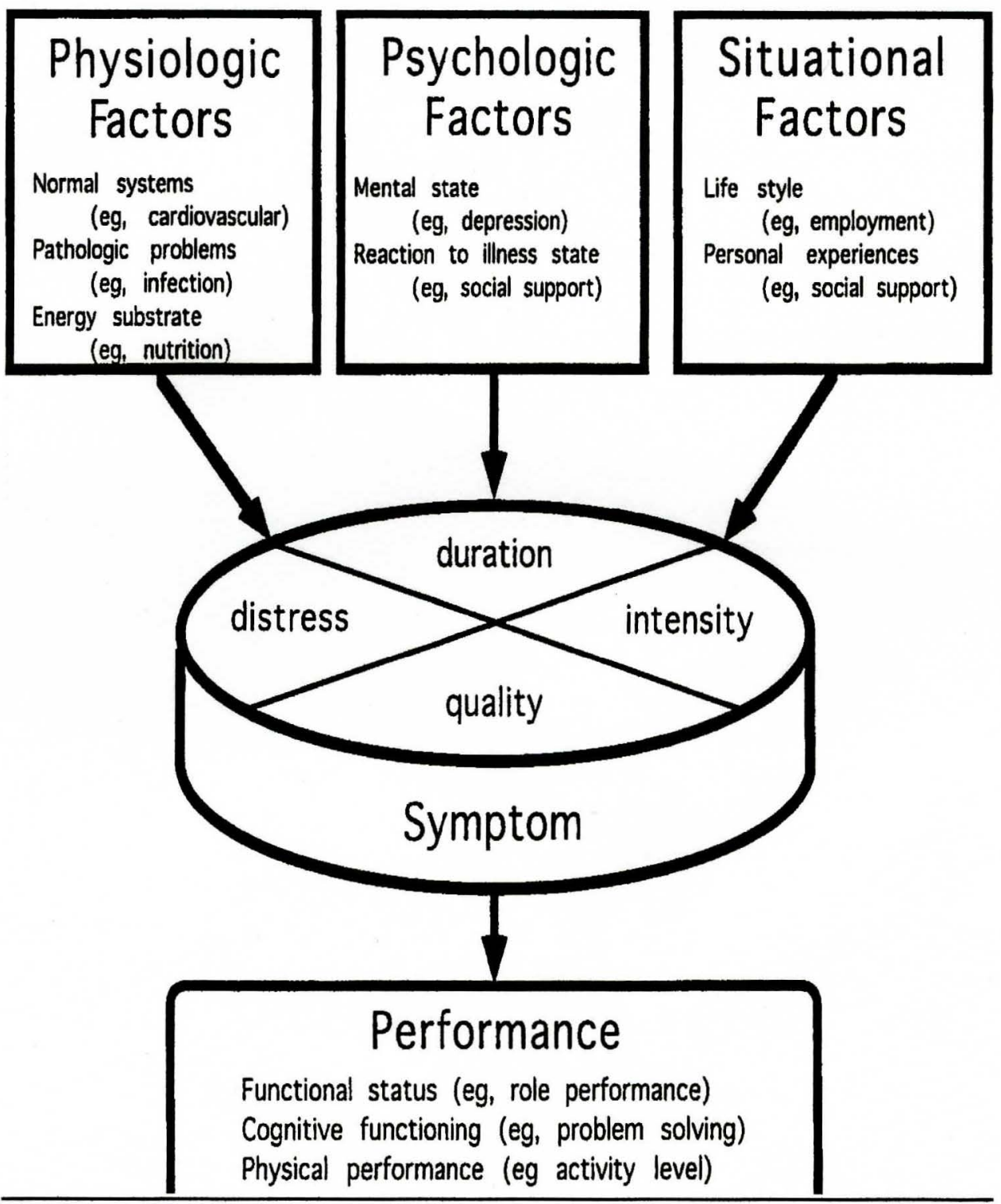

Figure 1. Theory of Unpleasant Symptoms 
clinical researchers. The assumption behind the theory is that there are sufficient commonalities among symptoms to warrant a theory that is not limited to one symptom (Lenz et al., 1997). The Theory of Unpleasant Symptoms has three major components: the symptoms that the individual is experiencing; influencing factors that give rise to or affect the nature of the symptom experience; and the consequences of the symptom experience (Lenz et al., 1997). Symptoms are conceptualized both individually and in combination with other symptoms. The theory asserts that more often multiple symptoms are experienced simultaneously. The Theory of Unpleasant Symptoms acknowledges that a symptom of given severity may be incapacitating to some clients, whereas it might be less bothersome to others (Sousa et al., 2011). Lenz et al. (1995) have argued that the theory is clinically applicable because it goes beyond symptomspecific concepts and theories. This theory stimulates thinking about common factors that may influence more than one symptom, the way in which multiple symptoms interact with one another and affect performance, and interventions that would mitigate multiple symptoms (Sousa et al., 2011).

Symptoms need to be assessed in terms of duration, intensity, distress, and quality. Influencing factors can be physiologic factors, psychological factors, and/or situational factors. Performance is described in terms of functional status, cognitive functioning, or physical performance. The Theory of Unpleasant Symptoms helps nurses recognize the need to assess multiple aspects of symptoms including characteristics, the underlying cause, as well as the frequency, intensity, duration, quality, and distress felt by the client due to the symptoms. This theory should stimulate nurses to consider factors 
that might influence more than one symptom and ways in which symptoms interact with each other (Lenz et al., 1997). This theory was useful in guiding this research, as constipation is an important client problem in CRC. This research paper identifies cases of constipation caused by the medication provided to prevent another symptom. With numerous interrelated symptoms potentially impacting client function, QOL, and other health-related outcomes, this theory helps the CNS identify the multidimensional nature of this problem. 


\section{Method}

\section{Purpose}

The purpose of this project was to determine the incidence of constipation in clients with CRC prescribed 5-HT3 inhibitors and to identify treatments that are successful in managing constipation while maintaining effective control of CINV.

\section{Research Questions}

The research questions were: What is the incidence of constipation in clients with colorectal cancer taking the 5-HT3 inhibitor, ondansetron? What treatments are successful in managing constipation in these clients while maintaining effective control of CINV?

\section{Design}

A retrospective descriptive study design was utilized.

\section{Site}

This study was conducted at The Leonard and Adele R. Decof Cancer Center located in the Fain Building at The Miriam Hospital, Providence, R.I. The Decof Center is a comprehensive cancer center providing care to clients with cancer and blood disorders.

\section{Sample}

The sample was recruited from the database of clients who had been treated at the Decof Cancer Center and who met inclusion criteria. Inclusion criteria included clients who completed chemotherapy consisting of Oxaliplatin or Irinotecan, Fluorouracil, and Leucovorin with ondansetron over a period of 24 months prior to the study and also 
experienced constipation as a result of receiving the antiemetic ondansetron. Clients who had been previously treated with chemotherapy were excluded, as well as clients with a documented history of constipation prior to receiving ondansetron, and those who did not receive ondansetron as part of the anti-nausea treatment for CRC..

\section{Procedure}

Prior to data collection activities, the proposal was reviewed and approved by the Lifespan and Rhode Island College IRBs. Institutional permission was obtained from the Director of Cancer Services and Ambulatory Care at the Decof Center. All active records are stored on site. The researcher obtained a list of clients who had been prescribed Oxaliplatin or Irinotecan, Fluorouracil and Leucovorin from the pharmacy. The list was then submitted to the Medical Records Department for record retrieval.

Records were first reviewed for eligibility requirements, and once determined data was collected using a researcher-developed data collection tool.

\section{Measurement}

A data collection tool was developed for this project. After IRB approval, the tool was pilot tested with three client records to assure completeness and usability. Data collected included: confirmed use of the drug ondansetron; whether the client was taking narcotics for pain management; age; the number of episodes of constipation; subjective complaints related to constipation; treatment to manage constipation including drugs prescribed to relieve constipation; and reported effectiveness of treatment. For purposes of this research, constipation was defined as having fewer than three bowel movements a week. Treatment for constipation was considered effective if the client had relief of 
constipation with resulting bowel movement within 24 hours of utilizing the prescribed treatment. 


\section{Results}

Eighty-two medical records were reviewed to obtain a cohort of 25 subjects requiring treatment for constipation. Four records were excluded due to documented history of constipation. All data obtained was stored in a locked file to which only the researcher and faculty advisor had access and confidentiality of all data was maintained.

Of the 82 records reviewed, 25 subjects were identified who had treatment for constipation. This result demonstrated a $32.8 \%$ rate of constipation. The reported risk of constipation related to the administration of ondansetron was 18\%-20\% (Up-To-Date).

The average age of subjects receiving treatment for constipation was 51.8 years, with a range of 33 to 86 years of age. Although documentation addressing constipation was retrieved, the subjects' description of the effectiveness of treatment for constipation was absent. Thee average age of the subject reporting a complaint of constipation was 70.4 years with a range of 31 to 76 years of age. The average age of the subject experiencing constipation was thus significantly higher than the average age of the cohort of subjects (51.8years).

Twenty-one of the 25 subjects reported one episode of constipation (84\%), with an average age of 57.3 years old. Two of the 25 subjects ( $8 \%$ ) reported two and three episodes of constipation each. Twenty-one of 82 subjects ( $26 \%$ ) were noted to have been prescribed narcotics for pain management. In those records, it was noted by the clinician that the constipation experienced by the subject was not related to the narcotic use, but to the ondansetron. 
The treatment of constipation was varied, as can be seen from Figure 2.

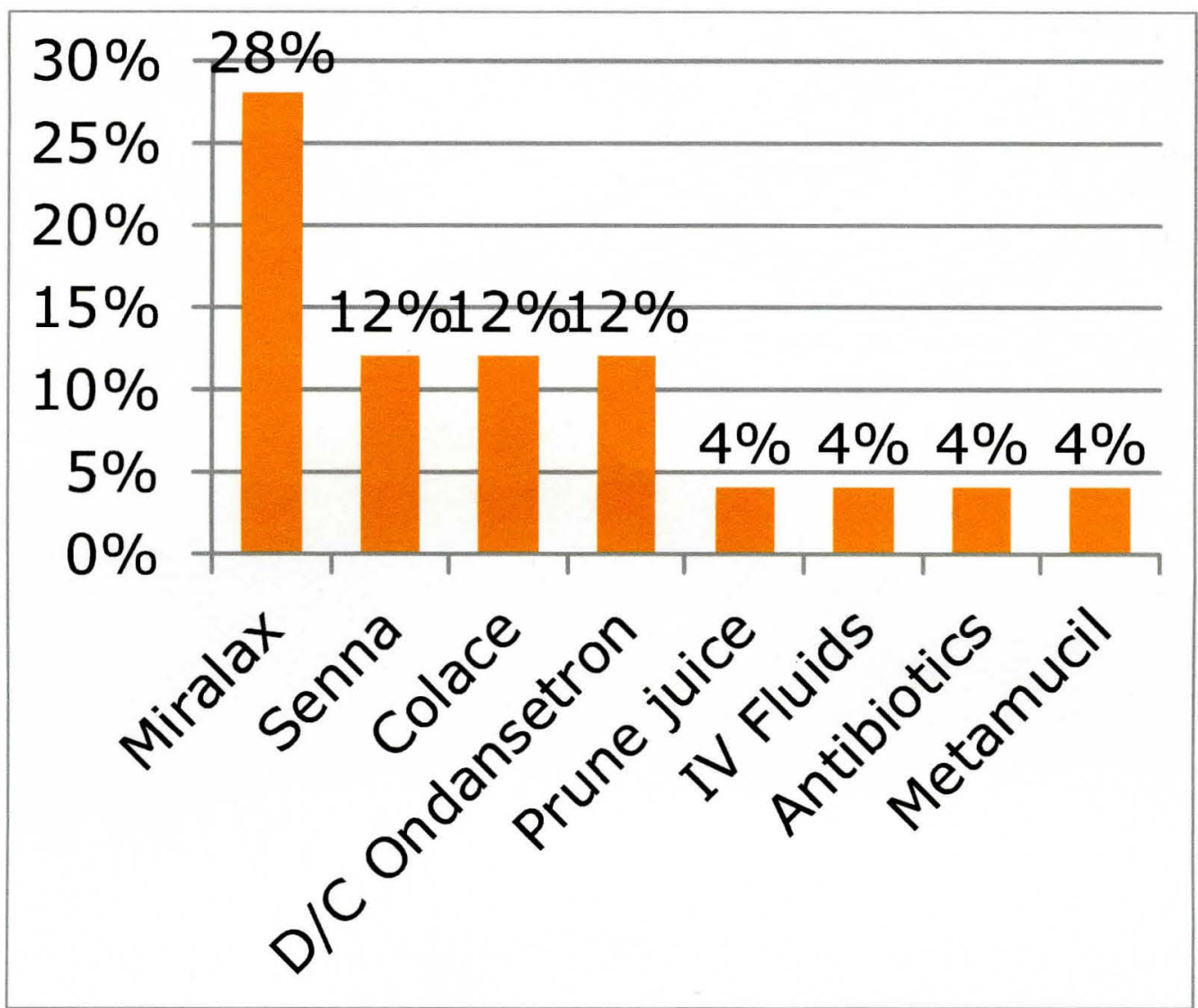

Figure 2: Medications providing effective relief of constipation for subjects receiving ondansetron for the management of CINV.

The most frequently used medication was Miralax $(n=7)$, followed by Senna or Senokot $(n=3)$ and Colace $(n=2)$. The remaining treatments were described as follows: one subject took Metamucil, one received intravenous fluids, one received antibiotics for an infection, and one took prune juice. Six of the 25 subjects (24\%) experiencing constipation either had the ondansetron discontinued or the dose reduced. There was no relationship between the number of treatments a subject received and number of episodes 
of constipation reported. The researcher noted the episodes of constipation were reported at varying times throughout the client's chemotherapeutic cycle. 


\section{Summary and Conclusions}

Constipation is a general term used to describe the difficulties a client experiences with moving his or her bowels. There is no universally accepted definition of constipation (Heitkemper \& Wolff, 2007). Constipation is a problem of increased significance with cancer in general, and for those with CRC in particular. The purpose of this project was to determine the incidence of constipation in clients with CRC prescribed the 5-HT3 inhibitor ondansetron and to identify treatments that are successful in managing constipation while maintaining effective control of CINV. This study was guided by the Theory of Unpleasant symptoms, developed by Lenz et al. (1995; 1997), which specifies that the experience of symptoms is multidimensional and incorporates the potential interactions of multiple symptoms (Sousa et al., 2011).

A retrospective chart review was conducted; 82 charts were reviewed and 25 charts met the inclusion criteria. The data demonstrated a $32.8 \%$ rate of constipation, higher than the $18 \%-20 \%$ risk of constipation related to the administration of ondansetron that is reported in the literature (Up-To-Date). The treatment of constipation varied from client to client. The most frequently used medication was Miralax (28\%) followed by Senna or Senokot (12\%) and Colace $(8 \%)$.

This study was limited by factors associated with the retrospective design, in particular, by the fact that minimal documentation of client description of symptoms and treatment effectiveness was able to be identified. While 82 records were reviewed, the actual sample include 25 subjects. Gender had not been identified as a variable in the data collection tool and was unable to be retrieved. 
The CNS cares for and improves outcomes of clients through three spheres of influence: the client/family, nursing/nursing practice, and organizational/systems sphere (McKinley, 2007). While constipation is a commonly experienced symptom overall, in the CRC it is a significant contributor to decreased QOL. The CNS plays an important role in the management of this key symptom in the client with CRC. The CNS first conducts a comprehensive history to determine the etiology of the constipation, and then evaluates the client individually and determines whether diagnostic procedures are needed. Throughout the treatment process, the CNS assesses for adequate oral intake, determines medication use and compliance, performs abdominal and rectal exams and advises clients regarding effective therapeutic strategies. A comprehensive bowel regimen should be implemented at the initial chemotherapy treatment, with documentation of the effect of the regimen and recommendations as necessary. The choice of laxative should be discussed with each client individually, identifying any product the client found successful in the past. The clinician should avoid prescribing bulk laxatives in this fragile oncology population. Additionally, a comprehensive bowel regimen should be implemented at the initial chemotherapy treatment with documentation of the effect of the regimen and recommendations as necessary. The choice of laxative should be discussed with each client individually identifying any product the client found successful in the past. The clinician should avoid prescribing bulk laxatives in this fragile oncology population (Farjardo et al., 2006).

Though the sample was limited, based on the results of this study this writer will recommend that clients with a history of constipation not be routinely prescribed the 
antiemetic ondansetron, and that rather other effective antiemetics be identified in the antiemetic treatment plan. Further research is needed in this area in order to more broadly generalize results. Limited documentation of the subjects' description of symptoms along with minimal provider notes referring to the effectiveness of treatment was evident in the logs. This is an identified area for improvement, since this data is important when planning future treatments and optimizing symptoms management. 


\section{Implications and Recommendations}

As the treatment of the client with CRC continues to evolve, it is the role of the $\mathrm{CNS}$ as a member of the multidisciplinary team to continue to improve client outcomes by minimizing multidimensional symptoms. As identified in the Theory of Unpleasant Symptoms (Lenz et al., 1995; 1997), symptoms are conceptualized both individually and in combination with other symptoms, and each need to be assessed in terms of duration, intensity, distress, and quality. Influencing factors can be physiologic, psychological and/or situational factors, so it is essential that the treatment plan be broad and comprehensive. Addressing sources of distress and considering each individual client, especially when symptom management is not successful, is essential to an individualized, client-centered approach to care. Key principles are broad assessment for symptoms and other sources of distress and prioritizing which symptoms are most affecting client's QOL (Dy \& Apostol, 2010).

The CNS plays a pivotal role in directing the care of the client with cancer, at the individual, institutional, local, and national levels. Advocacy for continued treatment advancements and research is critically needed. Continued research related to symptom management is needed overall and in the CRC population. In particular, constipation is a commonly experienced symptom in this group that is often minimized in practice and yet adversely affects QOL. Research should continue to explore strategies to improve QOL in this population and to better manage symptoms such as constipation that also threaten on-going compliance with chemotherapy regimens. While this project focused on the impact of one drug on constipation, further research related to contributors to this 
symptom and interventions, including evidence-based holistic regimens, should be explored. Research with diverse subjects, representative of the CRC population, is indicated.

The CNS has the potential to play a leadership role in the management of clients with CRC. The literature is robust related to management of nausea and vomiting associated with chemotherapy regimens; further work is needed related to management of the symptom of constipation. 
References

American Cancer Society, Colorectal cancer facts \& figures 2011-2013. Atlanta: American Cancer Society, 2011.

Andreyev, H.J.N., Davidson, S.E., Gillespie, C., Allum, W., \& Swarbrick, E., (2012). Practice guidance on the management of acute and chronic gastrointestinal problems arising as a result of treatment for cancer. Gut Journal, 61 . doi:10.1136/gutjnl-2011-300563.

Arcangelo, V.P., \& Peterson, A.M., (2006). Pharmacotherapeutices for advanced practice: a practical approach. New York: Lippincott, Williams, \& Wilkins.

Dy, S.M., \& Apostol, C.C., 2010. Evidenced approaches to other symptoms in advanced cancer. Cancer Journal, 16(5), 507-513.

Farjardo, N.R., Cremonini, F., \& Talley, N., (2006). Management of constipation in patients with cancer. American Journal of Cancer, 5(5), 319-330.

Fulton, J.S., Lyon, B.L., \& Goudreau, K.A. (2010). Clinical nurse specialist practice. New York. Springer Publishing Company. pp. 61-62.

Hanson, C.M., \& Spross, J.A. (2005). Advanced practice nursing, 4th Edition: an integrative approach. St. Louis, MO: Saunders.

Heitkemper, M., \&Wolff, J., (2007). Challenges in chronic constipation. The Nurse Practitioner, 32(4), 36-42.

Jefford, M., Lotfi-Jam, K., Baravelli, C., Grogan, S., Rogers, M., Krishnasamy, M., \& Schofield, P. (2011). Development and pilot testing of a nurse-led post treatment support package for bowel cancer survivors. Cancer Nursing, 34(3), E1-E9. 
Kim, M.W., (2011). Effects of oncology clinical nurse specialists' interventions on nursing-sensitive outcomes in South Korea. Clinical Journal of Oncology Nursing. 15(5), E66-E74.

Lenz, E.R., Suppa, F., Gift, A.G., Pugh, L.C., \& Milligan, R.A. (1995). Collaborative development of middle-range nursing theories: toward a theory of unpleasant symptoms. Advances in Nursing Science, 17/3, 1-13.

Lenz, E.R., Suppa, F., Gift[me1][me2][me3], A.G., Pugh, L.C., \& Milligan, R.A. (1997). The middle-range theory of unpleasant symptoms: an update. Advances in Nursing Science, 17(3), 14-27.

Librach, S.L., Bouvette, M., DeAngelis, C., \& Pereira, J.L., (2010). Consensus recommendations for the management of constipation in patients with advanced, progressive illness. Journal of Pain and Symptom Management, 40(5), 761-773.

Likun, Z., Xiang, J., Yi, B., Xin, D., \& Tao, Z.L. (2011). A systematic review and metaanalysis of intravenous palonosetronin the prevention of chemotherapy-induced nausea and vomiting in adults. The Oncologist, 16, 207-216.

Lohr, L., (2008). Chemotherapy induced nausea and vomiting. The Cancer Journal, 14(2), 85-93.

McKinley, M.G., (2007). Acute and critical care clinical nurse specialists: synergy for best practices. St. Louis, MO: Saunders.

Miakowski, C., (2011). Patients' stories provide insight. Cancer Nursing, 34(4), 340.

Nagaviroj, K., Yong, W.C., Fassbender, K, Zhu, G., \& Onechuk, D. (2011). Comparison of the constipation assessment scale and plain abdominal radiography in the 
assessment of constipation in advanced cancer patients. Journal of Pain and Symptom Management. 10.1016/j.jpainsynmman.2010.11.019

Navari, R.M. (March 26, 2009). Pharmacological management of chemotherapyinduced nausea and vomiting: Focus on recent Developments. Drugs: 69(5), $515-533$.

National Comprehensive Cancer Network Clinical Practice Guidelines in Oncology, 2011.

Pollock, J., \& Welsh, J.S., (2011). Clinical cancer genetics, part 1. American Journal of Clinical Oncology, 34(3), 332-336.

Rangwala, R., Zafar, Y., \& Abernethy, A.P., (2012). Gastrointestinal symptoms in cancer patients with advanced disease: new methodologies, insights, and a proposed approach. Current Opinion Supportive Palliative Care, 6(1), 69-76. 10.1097/SPC.0b013e32834f689d

Rao, S., \& Go, J.T. (2010). Update on the management of constipation in the elderly: new treatment options. Clinical Interventions in the Elderly, 5. 163-171.

Saif, M.W., \& Chu, E (2010). Biology of colorectal cancer. The Cancer Journal, 16/3, 196-201.

Shafi, M.A., \& Bresalier, R.S. (2010). The gastrointestinal complications of oncologic therapy. Gastrointestinal Clinic of North America, 39, 629-647.

Sousa, K.H., McDermott, C.M., \& Weiss, J., (2011). The importance of assessing symptoms and symptom clusters in critical care units. American Association of Critical-Care Nurses, 22(3), 275-281. doi: 10.1097/NCI.0b013e3182209279 
Viale, P. H. (2006) Update on the management of chemotherapy-induced nausea and vomiting. Journal of Infusion Nursing, 29(5), 283-292.

Wilson, T.R., Alexander, D.J., Kind, P., \& Phil, M. (2006). Measurement of healthrelated quality of life in the early follow-up of colon and rectal cancer. The American Society of Colon and Rectal Surgeons, 49(11). 1692-1702.

Up-To-Date

www.Americancancersociety.org

www.cancer.org/research/cancerfactsfigures.

www.phpartners.org/healthstatshtml 